\title{
Perpetuation of Leishmania: some novel insight into elegant developmental programs
}

\author{
Geneviève MiLON* \\ Institut Pasteur, Département de Parasitologie et Mycologie, Unité Immunophysiologie et Parasitisme \\ Intracellulaire, 25 rue du Dr Roux, 75724 Paris Cedex 15, France
}

(Received 9 October 2008; accepted 16 April 2009)

\begin{abstract}
Leishmania spp. are polarized single-celled eukaryotic parasites, the perpetuation of which relies on two other organisms they "use" as hosts. One of the Leishmania host organisms is a blood-feeding female sand fly, the second host being a mammal that acts as a blood source for the female sand fly. Leishmania-hosting sand flies transmit the metacyclic promastigote developmental stage to the mammal skin. While many mammals are known to act as sand fly blood sources, only some of these mammals are/ will be "used" as Leishmania hosts. This host status means that skin as well as skin-distant tissues and cell lineages (mononuclear phagocytes and fibroblasts) of these mammals are rapidly and continuously remodelled as niches where Leishmania will deploy its developmental programs: it is noteworthy that without the deployment of the developmental program underlying Leishmania transmission from the mammal to the blood-searching and blood-feeding sand flies, the perpetuation of Leishmania will be suspended. While post genomic approaches are providing insight about some features of Leishmania major, Leishmania infantum/chagasi and Leishmania braziliensis, such approaches are not yet available for the natural hosts (wild rodents, wild sand flies) these Leishmania species "use" as hosts.
\end{abstract}

parasitism / tissue and cell remodeling / parasite transmission

\section{Table of contents}

1. Introduction.....

2. The relevant features of the living organisms Leishmania spp. "use" as hosts in their natural ecosystems

2.1. The example of the natural hosts of L. major, the "best known/less neglected" Leishmania species with respect to laboratory and field research

2.2. Compared to the progressively built knowledge about the L. major natural hosts what do we know about the natural hosts of the other Leishmania species?

3. The relevant features of the female sand flies when they are "used" as Leishmania hosts and as Leishmania vectors i.e. when they are transmitting the metacyclic promastigotes in the mammal dermis

4. The relevant features of mammals when their skin is hosting Leishmania amastigotes that are transmissible to sand flies

4.1. The mammal skin: a key compartment with respect to (a) sand fly' blood-feeding and

(b) to Leishmania establishment and persistence whatever the Leishmania species

\footnotetext{
* Corresponding author: genevieve.milon@pasteur.fr
} 
4.2. Leishmania persistence in the mammal skin and its transmission to the sand flies: how rapidly is this developmental phase reached in stable ecosystem(s) and could it be modelled?

5. Concluding remarks and perspectives.

\section{INTRODUCTION}

This brief contribution presents the current understanding of some of the stepwise processes the single-celled protozoan parasites named Leishmania "use" to ensure their perpetuation in host organisms. I have attempted to highlight the key processes on which the transmission of Leishmania parasites depends: (a) from bloodfeeding sand flies to mammals and (b) from mammals to blood-feeding sand flies. Although Leishmania were first recognized as etiological agents of human infectious diseases (known as visceral, cutaneous or muco-cutaneous leishmaniasis [5, 18, 38]), nowadays Leishmania spp. are also studied as living parasites deploying elegant developmental programs in the two different organisms they "use" as hosts [7, 37, 56]. As briefly introduced above, one of the two Leishmania-hosting populations is a bloodfeeding sand fly population of limited size with respect to the whole sand fly population and the other Leishmania-hosting populations are mammal populations, which first act as blood donors for the blood-feeding female sand flies. The mammals are (a) non-human mammals such as wild rodents (e.g. Meriones, Psammomys, Rhombomys exploited as hosts by Leishmania major, e.g. Proechymis exploited as host by Leishmania amazonensis) (b) humans, being accidental hosts for most of the Leishmania species, or (c) only humans (e.g. Leishmania donovani stricto sensu). Thus, the sand flies and sand fly blood donors experience a unique relationship, the latter allowing the sand flies to produce their progenies [28, 52].

The knowledge reviewed within the present contribution has been acquired within the frame of this/these complex and dynamic ecosystem(s), but it is far from covering all the issues especially those which could result in the spreading of Leishmania species hosted by anthropophilic sand flies [3, 20]. My main objective is to invite every investigator whatever her/his initial training (basic life sciences and/or biomedical sciences) to capture the essence of parasitism, which means to study the Leishmania parasites as living organisms per se, instead of only studying them as "human pathogens" [37]. I think that the conceptual frame I am promoting will allow further investigation into the processes essential for Leishmania spp. perpetuation, whatever the level of analysis - molecular, sub-cellular, cell, tissue, individual host organisms and/or host populations.

\section{THE RELEVANT FEATURES OF THE LIVING ORGANISMS LEISHMANIA SPP. "USE" AS HOSTS IN THEIR NATURAL ECOSYSTEMS}

\subsection{The example of the natural hosts of $L$. major, the "best known/less neglected" Leishmania species with respect to laboratory and field research}

Understanding the main features of the processes that account for the perpetuation of the organisms (sand flies and rodents) that are subverted as hosts by $L$. major will enable features of the natural ecosystem(s), where these three partners are experiencing biological partnerships, to be assessed $[22,23]$. It is noteworthy that in stable ecosystems the development of L. major in wild rodents does not result in skin damage - i.e. there are no symptoms of localized cutaneous leishmaniasis, a major difference with the outcome in humans who are more or less transiently exposed within this natural and stable ecosystem. Indeed, in natural settings (nearly free of any human beings) successful sand fly development involves their complex relationships with the rodents of one of three genera - Meriones, Psammomys, 
Rhombomys. Briefly, the male and female sand flies reach the adult developmental stage if trophic and non trophic resources are available for the pre-adult developmental stages, which are known to be terrestrial $[1,30-32,52]$. The pre-adult sand fly developmental stages do develop within the habitat of the rodents, benefiting from plant remnants brought by the rodents as well as from rodent faeces. At the adult developmental stage, both male and female sand flies feed on plants, organisms from which they capture sugars. The female sand flies regularly search for and more or less successfully take blood meals most frequently from rodents, with only a proportion of those mammals being exploited as Leishmania hosts. It is noteworthy that, some of the rodents such as Psammomys (acting as sand fly blood donors) rely on one plant genus/species. These features have led to original interventions such as the disruption of the plant habitat, the latter resulting in the disruption of both the rodent and sand fly population habitats. Such a strategy was indeed assayed in Tunisia in a L. major focus at the periphery of a village where the human population has been extending its activities and habitat ${ }^{1}$.

\subsection{Compared to the progressively built} knowledge about the $L$. major natural hosts what do we know about the natural hosts of the other Leishmania species?

For L. amazonensis or L. (Viannia) species, the natural ecosystems on which the host perpetuation relies upon are located in the Amazonian forest. Among the few sustained investigations which addressed the features of natural ecosystems where species other than L. major have perpetuated for millions of years $[4,10,16,24,25,41,56]$ those deployed in French Guiana are still a reference $[17,49]$. In this latter case, the most reasonable way for humans to avoid becoming a sand fly blood source and a parasite host is again to avoid activities in the Amazonian forest, especially when the female sand flies are actively searching for their blood meal. Sorting out the features

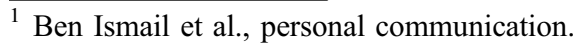

of the hosts of L. chagasi [21] and of L. donovani stricto sensu with respect to their natural ecosystems illustrate the following points: in regions such as India and East Africa where the parasites were originally detected as etiological agents of visceral leishmaniasis, humans are still the main mammals who act as both blood sources of the sand flies as well as L. donovani hosts. Most frequently, the L. donovani development and persistence do not result in local or systemic damage in a high proportion of any human population inhabiting stable ecosystems. The density of $L$. donovanifree as well as $L$. donovani-hosting sand flies, and the nature of their resting habitat (endophilic versus exophilic) are important parameters to determine in time and space if we seriously wish to use insect-targeting interventions to reduce the parasite delivery to humans [29-32, 41, 58, 59, 63, 64]. As far as L. tropica is concerned, the investigations performed by Volf and colleagues [60] will provide insight on the features of the natural ecosystems this Leishmania species is relying on.

\section{THE RELEVANT FEATURES \\ OF THE FEMALE SAND FLIES WHEN \\ THEY ARE "USED” AS LEISHMANIA \\ HOSTS AND AS LEISHMANIA VECTORS \\ I.E. WHEN THEY ARE TRANSMITTING \\ THE METACYCLIC PROMASTIGOTES \\ IN THE MAMMAL DERMIS}

Sorting out the different trophic and non trophic signals which operate at the onset of (i) the gonotrophic cycle and (ii) the Leishmania developmental program will benefit from combined in vitro approaches that allow axenic parasite life traits to be characterized and in vivo approaches such as RNA interference technology applied to the blood-feeding insects [19, 53]. With such approaches, it will be possible, very soon, to document the respective contribution of the sugar and/or blood meals when the female sand flies are exploited as Leishmania hosts. The Leishmania developmental program is restricted to one tissue of the blood-feeding sand flies, namely the mid gut. Parasitism in the adult female sand fly is initiated when it 
takes blood from a mammal, the dermis of which is a protected niche of phagocytic leucocytes or fibroblasts hosting insect' pre-adapted amastigotes (see Sect. 4). The blood mealloaded with intracellular Leishmania amastigotes is delivered to the posterior mid gut, a compartment from which the blood-derived nutrients are extracted and from which the non trophic signaling molecules that contribute to the onset and progression of the insect progeny production are generated [52, 65, 66]. Thereafter, Leishmania amastigotes rapidly exit the mammal cells and differentiate as procyclic promastigotes with very short flagella. Many distinct developmental stages are now known to proceed as the promastigotes migrate from the posterior mid gut to the anterior mid gut where the stomodeal valve is located, which is the boundary between the anterior mid gut and the foregut and a component of the pump system used in blood feeding [52, 62, 66]. Briefly, the first promastigote multiplication phase takes place in the early blood meal confined by the sand fly-derived peritrophic matrix. Promastigotes develop into large slender forms which (a) traverse the peritrophic membrane, (b) anchor themselves to the epithelial cells lining the insect posterior mid gut and (c) undergo the second multiplication phase before (d) migrating to the anterior part of the gut. Though two stages are observed at the stomodeal valve, haptomonads and metacyclic promastigotes, only the latter (known to have exited the cell-cycle) are found upstream and downstream of the stomodeal valve and are highly adapted for successful transmission to the mammalian host. The stomodeal valve is a key location for the Leishmania promastigote developmental stages: indeed while they are embedded in a gel they secrete, they are prevented from reaching the foregut. Those that escape being embedded in the gel reach the foregut downstream from this valve $[8,9,27-$ $33,35,36,44-47,50,51,54,56-58,62]$. Therefore, could any differences in bloodfeeding efficiency result in differences in (i) the number and (ii) the conditions of the intra-dermal delivery of metacyclic promastigotes if the sand flies were hosting such mammal-infective Leishmania developmental stages? Considering the complexity of the metacyclogenesis process, at least two of the following scenarios are plausible. Those metacyclic promastigotes that have reached the pharynx, the cibarium and the proboscis i.e. the most anterior part of the sand fly' digestive tract, are expected to be deposited in low numbers during the probing phase when the female is locating the most relevant place where to take its blood meal. If the sand fly female does create the blood pool, the metacyclic promastigotes embedded in the gel are expected to be regurgitated together with the gel in which case a higher number of Leishmania will be delivered, the more rapid bona fide metacyclics reaching the resident macrophages or the monocytes that are starting the differentiation process while attaching and spreading on the dermal extra-cellular matrix (ECM) surrounding the blood pool remnant. Recently a very interesting and carefully performed analysis [33] provided the first estimates of $L$. major promastigotes delivered by the Phlebotomus dubosqi sand fly vector to the mouse ear skin. Using a realtime PCR based-approach optimized to detect as few as $10 \mathrm{~L}$. major promastigotes deposited into the mouse ear dermis, Kimblin et al. [33] were able to document a remarkable range (10-10 000) in the dose of L. major transmitted to mice exposed to a single $L$. major-hosting P. dubosqi. The majority (75\%) of the transmitting sand flies delivered $\sim 600$ or fewer promastigotes, and the remainder (25\%) delivered 1000 or more promastigotes. Among the transmitting flies, the investigators note a set of parameters assessing their ability to deliver a high versus low dose of promastigotes. The parameters predictive of high-dose transmission are the following: (a) pre-feeding promastigote load value of 30000 ; (b) an inability to fully engorge since a relatively high proportion of the promastigotes, otherwise shown to be located in the anterior part of the mid gut, being egested. Each of these parameters is consistent with the observations that sand flies with a high parasite load embedded in a parasite-derived gel - known as Parasite-secreted Gel (PSG) do experience difficulty in feeding: indeed, the 
stomodeal valve is forced open by the pressure of the plug and/or by direct damage to the valve as a consequence of a chitinase secreted by attached promastigotes [48]. From these data, it is more than likely that an especially huge promastigote load in the most anterior part of the mid gut will promote the conditions under which an important fraction of the promastigotes can "become mobilized" in the mammal dermis, through regurgitation, at the point of blood vessel disruption i.e. where the blood pool will be captured by the sand fly. These occasional "high-dose promastigote deliveries" consolidate one of the former conclusions formulated by Rogers et al. [46]: briefly, Rogers et al. consider that the delivery in the mammal dermis of high numbers of promastigotes cannot be accounted for by parasites having reached the foregut - i.e. the compartment downstream of the stomodeal valve - where they are expected to move freely at low numbers; but only through regurgitation of parasites embedded in the PSG plug. Thus, the major sources of low-dose inoculum is expected to be sand flies that experience interrupted feeding "abandoning their blood-feeding attempts" or that ultimately succeed in blood uptake without "dislodging" the promastigotes embedded in the gel/plug upstream of the stomodeal valve.

The best estimate before the Kimblin et al. analysis [33] was reported by Rogers et al. [46] at $10^{3}$ per biting fly. In their study, the dose delivered by groups of Lutzomyia longipalpis sand flies hosting Leishmania mexicana (a permissive though not natural Leishmania/sand fly combination) was calculated as the average number of promastigotes collected in the culture medium per fly allowed to feed through the artificial membrane, an approach that did not allow capturing and estimating the variation in the metacyclic promastigote numbers delivered by individual flies. While comparing their study to the study of Rogers et al. [46], Kimblin et al. [33] raise the questions of the potential alterations in the behaviour of sand fly feeding on an artificial membrane rather than a living mammal - in their case anesthetized mice known to be a parasite-supportive host. Further studies will provide answers to these questions.
4. THE RELEVANT FEATURES OF MAMMALS WHEN THEIR SKIN IS HOSTING LEISHMANIA AMASTIGOTES THAT ARE TRANSMISSIBLE TO SAND FLIES

\subsection{The mammal skin: a key compartment with respect to (a) sand fly' blood-feeding and (b) to Leishmania establishment and persistence whatever the Leishmania species}

Now I turn mainly to rodent and human mammals, starting with a brief description of the features of mammal skin, its draining lymph node and the vascular beds, the lymphatic bed connecting the skin to the skin-draining lymph node a tissue known as a secondary lymphoid organ [26, 34, 55]. The skin surface displays three anatomic layers: (a) the avascular epidermis, (b) the dermis and (c) the sub-cutis which serve independent as well as coordinated functions. Like other tissues, the sub-epidermal skin compartment displays two major circulatory systems: the blood and lymphatic vascular beds. Both vascular beds play complementary functions in tissue perfusion and fluid circulation. Before rapidly highlighting the mammal skin compartments, and its blood vascular bed, let me share our current understanding of the structure and function of the lymphatic vascular system. From the upper dermis, lymphatic capillaries drain lymph - i.e. interstitial fluid composed of both small and large molecules and bathing the ECM and the cell lineages fibroblasts, mononuclear phagocytes, mast cells-anchored to the ECM. The lymph is drained to the skin-draining lymph node by a system of blind-ended vessels lined by lymphatic endothelial cells (LEC). While most dermal lymphatic capillaries remain collapsed in a steady state, when interstitial pressure increases, the anchoring filaments (known to connect LEC to the ECM) exert tension on these LEC allowing the interstitial fluid to enter the opened lumen of the lymphatic capillaries. The afferent lymphatic vessels drain into the skin-draining lymph node sub-capsular sinus, distal to the hilum $[26,61]$. 
The vessel-free epidermis is separated by a basement membrane from the dermis. The latter is known to be composed of two compartments, the upper papillary dermis and the deeper reticular dermis. Human skin displays several functionally distinct vascular units such as the following: (a) the capillary loops within the tip of the papillae, (b) the superficial vascular plexus (SVP), (c) the deep vascular plexus (DVP) and (d) the subcutaneous plexus. In the skin as in other organs, blood capillaries are the terminal segment of the arterial tree and connect with the venular capillaries, which empty out in the post-capillary venules, the latter being surrounded by two to three layers of pericytes. Due to the small size of the adult female sand flies and the close proximity of the capillary loop and SVP endothelial cells to the epidermis, all the processes underlying the blood-searching and blood-feeding processes displayed by the sand flies are expected to rely on the epidermis-dermis communication. Though laboratory mice are relevant mammals for sorting out many features of this epidermal-dermal communication, some of the features of the latter are expected to be distinct from those in humans: indeed the mouse dermis lacks capillary loops and no clear distinction exists between the SVP and DVP. These differences justify the recourse to humanized mice for further investigation [42].

\subsection{Leishmania persistence in the mammal skin and its transmission to the sand flies: how rapidly is this developmental phase reached in stable ecosystem(s) and could it be modelled?}

This brief description of the skin compartments from which the sand flies are expected to take their blood highlights that the blood feeding habit is a complex process, which can be divided into at least two phases (a) the probing phase and (b) the blood recovery phase. It is important to refine our understanding of the mammalian blood donor's constitutive and/or inducible processes which result in the most efficient blood capture, comparing mammals that have never been exposed to blood-feeding arthropods to those that have been previously exposed. At least for the blood-feeding sand fly females, it was documented that bloodfeeding is more efficient in mammals preexposed to saliva than in mammals which were never exposed to sand flies [11]. The severe combined immune-deficient (SCID) mice grafted with either superficial or deep human skin compartment will provide more relevant conditions for exploration of the sand fly blood-feeding processes from the probing phase to the blood meal per se in both naïve and saliva-sensitized humanized mice [42].

If properly considered, all these complex features of the skin and its draining lymph node (DLN) will impact our understanding of processes that assess the "living together of the three partners". Indeed both the sand fly' blood feeding processes per se and the early and late Leishmania developmental programs taking place in the Leishmania-loaded skin are expected to be highlighted. Briefly, as far as the early processes are concerned, the skin repair processes proceeding at the site of acute and transient skin wound created by the blood-feeding Leishmania-hosting sand fly could vary according to both (i) the components-saliva and PSG-co-delivered with the promastigotes and (ii) the mammal genetic makeup [6]. The early processes could condition the further developmental program of Leishmania amastigotes: either the location/ restricted expansion of the intracellular amastigote population will result in the sustained development of a balanced population of Leishmania-reactive effector and regulator $\mathrm{T}$ lymphocytes preventing any skin damage to deploy; or the location/unrestricted expansion of the intracellular amastigote population will result in a second and more prolonged skin damage [12-14, 46] that will also proceed towards spontaneous healing. It is noteworthy that the key questions of the coupling of (i) skin damage healing, (ii) amastigote persistence and (iii) transmissibility to the sand flies will be further addressed in the last section of this brief review. But before this, I wish to address how multi-parametric analysis will help sort out the key features of the stepwise developmental 
program of Leishmania, the program relevant to their transmissibility to the sand fly being an essential one. Indeed the simultaneous monitoring, in real time, of several parasite and host biomarkers, at the mouse tissue level, will provide a more comprehensive picture of Leishmania - and host - driven processes. In the present contribution only one question will be further addressed, namely, the question aimed at deciphering when and where the Leishmania developmental program allowing persisting amastigotes to be transmitted from the mammal to the sand fly is or will be switched on. Together with Belkaid [12], I pioneered laboratory mouse-based models that allow relevant questions to be addressed by selecting the mouse ear dermis as a site of Leishmania promastigote intra dermal delivery, the main objective being to easily follow the processes from the time when a few parasites are delivered to the mammal dermis to the stage of parasite transmission to the next host i.e. the sand fly $[33,37]$. At the early phase of the assay designed together with Sacks and Belkaid, we considered that transmission of a low number $(N \leq 100)$ of metacyclic promastigotes would occur when the sand fly would probe the skin for locating the small vessels it is actively searching for [12]. Later, when considering the data of Rogers et al. [46], a higher number $\left(N \leq 10^{4}\right)$ of metacyclic promastigotes was delivered in the ear dermis of mice of different inbred strains, the metacyclic promastigotes being transgenic luciferase-expressing Leishmania. Briefly, firefly luciferase-expressing L. major or $L$. amazonensis were generated and inoculated in the ear dermis of mice [37]. Naïve C57BL/6 mice display the status of a relevant rodent host to address the key question I introduced above [37]. Briefly, in C57BL/6 at day 50 post inoculation of $10^{4}$ L. major metacyclic promastigotes, the inoculated ear is hosting an intracellular amastigote population of low and stable size, this population influencing the deployment of balanced dynamic processes to which many leukocyte populations do contribute, with the driving ones, at this stage, being parasite-reactive regulatory [67] and effector T lymphocytes [12-14]. The cell lineage(s) that hosts this stable amastigote population still need(s) to be identified and characterized not only within the primary inoculation site $[15$, $37,39]$, but also in other distant skin sites reached by amasitgote-shuttling cells [40]. In addition, the outcome of the parasite progenies (amplitude and kinetics of the amastigote population size fluctuations) post promastigote delivery by metacyclic-hosting sand flies is expected to depend upon the former exposure of the mammal rodents to which they are delivered [11]. Briefly, not only the presence of salivareactive $\mathrm{T}$ lymphocytes are expected to influence these parameters but also the presence of T lymphocytes reactive to Leishmania PPG i.e. the major component of the PSG - and to Leishmania-derived peptides are expected to operate in rodents previously exposed respectively to Leishmania-free sand flies or to Leishmania-hosting sand flies. I expect that in mice previously exposed to saliva, PSG and Leishmania, a second inoculum of low size delivered by metacyclic promastigote-hosting and probing sand flies will result in an accelerated remodeling of the skin and its DLN that will allow the amastigotes to rapidly reach the features of being transmissible to the sand flies.

Considering the wide parasite dose range transmitted by single sand flies, it will be important to know how this will be translated (a) not only with respect to the early skin damage onset, amplitude and duration, at the parasite inoculation point by the sand fly which regurgitates the Leishmania inoculum (b) but also with respect to the status of "persisting Leishmania" - hosting mice that act as sources of amastigotes transmissible to the sand flies. To address this important question an assay - designed to mimic transmission by sand flies - was recently set up by Sacks et al. [33]: C57BL/6 mice were handled in such a way that one of their ears was exposed to five parasite-free sand flies and either a "low" or "high" dose inoculum of Phleboto$m u s$ - derived L. major (Friedlin isolate) metacyclic promastigotes was given intra-dermally with a syringe in the sand fly exposed ears. Not only, clear differences in the progression of the otherwise transient ear damage initiated with a representative "high" or "low" 
dose inoculation were observed, but significant differences were noticed when the mice of the two groups were compared for their ability to act as long-term sources of amastigotes transmissible to the sand flies.

\section{CONCLUDING REMARKS AND PERSPECTIVES}

In conclusion, it is foreseeable that investigations on the developmental biology of Leishmania in its experimental hosts will unveil processes leading to more or less accelerated transmission from mammals to sand flies according to the physiological status of mammals, namely a host that was never exposed to either sand flies or to Leishmania and a host that was previously exposed to (i) either parasite-free sand fly saliva (ii) or to saliva co-delivered with a low metacyclic promastigote inoculum, (iii) or to Leishmania embedded in the parasite-derived gel. I hope to have provided the readers with an informative contribution about one of the most fascinating biological processes, namely perpetuation of Leishmania that rely on blood-feeding poolfeeders and mammals which first act as blood sources. Continual advances in our understanding of Leishmania life traits - including their transmission - should gradually shift our view of Leishmania from "pathogens" to living eukaryotic single-celled protozoan parasites displaying elegant developmental programs that are regulated over space and time. Probing a complex dynamic process such as parasite transmission indeed involves gathering spatial and temporal information within the most relevant experimental conditions, the latter being fed by rigorous in vitro studies [2, 39], real time imaging of the Leishmania - loaded skin at both the early [43] and late stages post the delivery of promastigotes by a single sand fly.

Acknowledgements. The Institut Pasteur, the Coordinated Action LEISHMED 6th Framework Programme/FP of the European Union, le Fonds dédié "Combattre les maladies parasitaires SanofiAventis, Ministère de la recherche et de l'enseignement supérieur" the FP7 LEISHDRUG consortium provided appraised funding. This manuscript is dedicated to the memory of Robert Desowitz and Frank Kunst two eminent biologists who inspired many of us who are unveiling the developmental programs of parasitic or commensal microbes in the organisms they use as hosts.

\section{REFERENCES}

[1] Adler S., Theodor O., The mouthparts, alimentary tract and salivary apparatus of the female in Phlebotomus papatasi, Ann. Trop. Med. Parasitol. (1926) 21:109-142.

[2] Antoine J.C., Prina E., Courret N., Lang T., Leishmania spp.: on the interactions they establish with antigen-presenting cells of their mammalian hosts, Adv. Parasitol. (2004) 58:1-68.

[3] Antoniou M., Haralambous C., Mazeris A., Pratlong F., Dedet J.P., Soteriadou K., Leishmania donovani leishmaniasis in Cyprus, Lancet Infect. Dis. (2008) 8:6-7.

[4] Arrivillaga J.C., Norris D.E., Feliciangeli M.D., Lanzaro G.C., Phylogeography of the neotropical sand fly Lutzomyia longipalpis inferred from mitochondrial DNA sequences, Infect. Genet. Evol. (2002) 2:83-95.

[5] Ashford R.W., The leishmaniases as emerging and reemerging zoonoses, Int. J. Parasitol. (2000) 30:1269-1281.

[6] Baldwin T., Sakthianandeswaren A., Curtis J.M., Kumar B., Smyth G.K., Foote S.J., Handman E., Wound healing response is a major contributor to the severity of cutaneous leishmaniasis in the ear model of infection, Parasite Immunol. (2007) 29:501-513.

[7] Bates P.A., Rogers M.E., New insights into the developmental biology and transmission mechanisms of Leishmania, Curr. Mol. Med. (2004) 4:601-609.

[8] Bates P.A., Transmission of Leishmania metacyclic promastigotes by phlebotomine sand flies, Int. J. Parasitol. (2007) 37:1097-1106.

[9] Bates P.A., Leishmania sand fly interaction: progress and challenges, Curr. Opin. Microbiol. (2008) 11:340-344.

[10] Beach R., Kiilu G., Leeuwenburg J., Modification of sand fly biting behavior by Leishmania leads to increased parasite transmission, Am. J. Trop. Med. Hyg. (1985) 34:278-282.

[11] Belkaid Y., Valenzuela J.G., Kamhawi S., Rowton E., Sacks D.L., Ribeiro J.M., Delayed-type hypersensitivity to Phlebotomus papatasi sand fly bite: an adaptive response induced by the fly? Proc. Natl. Acad. Sci. USA (2000) 97:6704-6709.

[12] Belkaid Y., Mendez S., Lira R., Kadambi N., Milon G., Sacks D., A natural model of Leishmania major infection reveals a prolonged "silent" phase of parasite amplification in the skin before the onset of lesion formation and immunity, J. Immunol. (2000) 165:969-977. 
[13] Belkaid Y., Piccirillo C.A., Mendez S., Shevach E.M., Sacks D.L., CD4+CD25+ regulatory $\mathrm{T}$ cells control Leishmania major persistence and immunity, Nature (2002) 420:502-507.

[14] Belkaid Y., Blank R.B., Suffia I., Natural regulatory $\mathrm{T}$ cells and parasites: a common quest for host homeostasis, Immunol. Rev. (2006) 212:287-300.

[15] Bogdan C., Mechanisms and consequences of persistence of intracellular pathogens: leishmaniasis as an example, Cell. Microbiol. (2008) 10:1134-1221.

[16] Bray D.P., Hamilton J.G., Host odor synergizes attraction of virgin female Lutzomyia longipalpis (Diptera: Psychodidae), J. Med. Entomol. (2007) 44:779-787.

[17] Dedet J.P., Pradinaud R., Gay F., Epidemiological aspects of human cutaneous leishmaniasis in French Guiana, Trans. R. Soc. Trop. Med. Hyg. (1989) 83:616-620.

[18] Desjeux P., Leishmaniasis: current situation and new perspectives, Comp. Immunol. Microbiol. Infect. Dis. (2004) 27:305-318.

[19] Dillon R.J., Ivens A.C., Churcher C., Holroyd N., Quail M.A., Rogers M.E., et al., Analysis of ESTs from Lutzomyia longipalpis sand flies and their contribution toward understanding the insect-parasite relationship, Genomics (2006) 88:831-840.

[20] Dujardin J.C., Campino L., Cañavate C., Dedet J.P., Gradoni L., Soteriadou K., et al., Spread of vector-borne diseases and neglect of Leishmaniasis, Europe, Emerg. Infect. Dis. (2008) 14:1013-1018.

[21] Feliciangeli M.D., Delgado O., Suarez B., Bravo A., Leishmania and sand flies: proximity to woodland as a risk factor for infection in a rural focus of visceral leishmaniasis in west central Venezuela, Trop. Med. Int. Health (2006) 11:1785-1791.

[22] Fichet-Calvet E., Jomâa I., Ben Ismail R., Ashford R.W., Leishmania major infection in the fat sand rat Psammomys obesus in Tunisia: interaction of host and parasite populations, Ann. Trop. Med. Parasitol. (2003) 97:593-603.

[23] Ginger M.L., Niche metabolism in parasitic protozoa, Philos. Trans. R. Soc. Lond. B Biol. Sci. (2006) 361:101-118.

[24] Ivens A.C., Peacock C.S., Worthey E.A., Murphy L., Aggarwal G., Berriman M., et al., The genome of the kinetoplastid parasite, Leishmania major, Science (2005) 309:342-436.

[25] Jochim R.C., Teixeira C.R., Laughinghouse A., Mu J., Oliveira F., Gomes R.B., et al., The midgut transcriptome of Lutzomyia longipalpis: comparative analysis of cDNA libraries from sugar-fed, blood-fed, post-digested and Leishmania infantum chagasiinfected sand flies, BMC Genomics (2008) 9:15.

[26] Johnson L.A., Jackson D.G., Cell traffic and the lymphatic endothelium, Ann. N.Y. Acad. Sci. (2008) 1131:119-133.
[27] Kamhawi S., The biological and immunomodulatory properties of sand fly saliva and its role in the establishment of Leishmania infections, Microbes Infect. (2000) 2:1765-1773.

[28] Kamhawi S., Phlebotomine sand flies and Leishmania parasites: friends or foes? Trends Parasitol. (2006) 22:439-445.

[29] Killick-Kendrick R., Leaney A.J., Ready P.D., Molyneux D.H., Leishmania in phlebotomid sandflies IV. The transmission of Leishmania mexicana amazonensis to hamsters by the bite of experimentally infected Lutzomyia longipalpis, Proc. R. Soc. Lond. B Biol. Sci. (1977) 196:105-115.

[30] Killick-Kendrick R., Biology of Leishmania in phlebotomine sandflies, in: Killick-Kendric R., Peters W. (Eds.), The Leishmaniases in Biology and Medicine, Academic Press, London, 1987, pp. 392-460.

[31] Killick-Kendrick R., The life-cycle of Leishmania in the sandfly with special reference to the form infective to the vertebrate host, Ann. Parasitol. Hum. Comp. (1990) 65:37-42.

[32] Killick-Kendrick R., Phlebotomine vectors of the leishmaniases: a review, Med. Vet. Entomol. (1990) $4: 1-24$

[33] Kimblin N., Peters N., Debrabant A., Secundino N., Egen J., Lawyer P., et al., Quantification of the infectious dose of Leishmania major transmitted to the skin by single sand flies, Proc. Natl. Acad. Sci. USA (2008) 105:10125-10130.

[34] Kupper T.S., Fuhlbrigge R.C., Immune surveillance in the skin: mechanisms and clinical consequences, Nat. Rev. Immunol. (2004) 4:211-222.

[35] Lainson R., Ryan L., Shaw J.J., Infective stages of Leishmania in the sandfly vector and some observations on the mechanism of transmission, Mem. Inst. Oswaldo Cruz (1987) 82:421-424.

[36] Lainson R., Rangel E.F., Lutzomyia longipalpis and the eco-epidemiology of American visceral leishmaniasis, with particular reference to Brazil: a review, Mem. Inst. Oswaldo Cruz (2005) 100:811-827.

[37] Milon G., Leishmania parasites: could we consider them as living organisms per se? Microbes Infect. (2008) 10:1077-1081.

[38] Murray H.W., Berman J.D., Davies C.R., Saravia N.G., Advances in leishmaniasis, Lancet (2005) 366:1561-1577.

[39] Osorio y Fortéa J., Prina E., de La Llave E., Lecoeur H., Lang T., Milon G., Unveiling pathways used by Leishmania amazonensis amastigotes to subvert macrophage function, Immunol. Rev. (2007) 219:66-74.

[40] Nicolas L., Sidjanski S., Colle J.H., Milon G., Leishmania major reaches distant cutaneous sites where it persists transiently while persisting durably in the primary dermal site and its draining lymph node: 
a study with laboratory mice, Infect. Immun. (2000) 68:6561-6566.

[41] Peacock C.S., Seeger K., Harris D., Murphy L., Ruiz J.C., Quail M.A., et al., Comparative genomic analysis of three Leishmania species that cause diverse human disease, Nat. Genet. (2007) 39:839-847.

[42] Pearson T., Greiner D.L., Shultz L.D., Humanized SCID mouse models for biomedical research, Curr. Top. Microbiol. Immunol. (2008) 324:25-51.

[43] Peters N.C., Egen J.G., Secundino N., Debrabant A., Kimblin N., Kamhawi S., et al., In vivo imaging reveals an essential role for neutrophils in leishmaniasis transmitted by sand flies, Science (2008) 321:970-974.

[44] Ready P.D., Leishmania manipulates sandfly feeding to enhance its transmission, Trends Parasitol. (2008) 24:151-153.

[45] Rogers M.E., Chance M.L., Bates P.A., The role of promastigote secretory gel in the origin and transmission of the infective stage of Leishmania mexicana by the sandfly Lutzomyia longipalpis, Parasitology (2002) 124:495-507.

[46] Rogers M.E., Ilg T., Nikolaev A.V., Ferguson M.A., Bates P.A., Transmission of cutaneous leishmaniasis by sand flies is enhanced by regurgitation of fPPG, Nature (2004) 430:463-467.

[47] Rogers M.E., Bates P.A., Leishmania manipulation of sand fly feeding behavior results in enhanced transmission, PLoS Pathog. (2007) 3:e91.

[48] Rogers M.E., Hajmová M., Joshi M.B., Sadlova J., Dwyer D.M., Volf P., Bates P.A., Leishmania chitinase facilitates colonization of sand fly vectors and enhances transmission to mice, Cell. Microbiol. (2008) 10:1363-1372.

[49] Rotureau B., Gaborit P., Issaly J., Carinci R., Fouque F., Carme B., Diversity and ecology of sand flies (Diptera: Psychodidae: Phlebotominae) in coastal French Guiana, Am. J. Trop. Med. Hyg. (2006) 75:62-69.

[50] Sacks D.L., Metacyclogenesis in Leishmania promastigotes, Exp. Parasitol. (1989) 69:100-103.

[51] Sacks D., Kamhawi S., Molecular aspects of parasite-vector and vector-host interactions in leishmaniasis, Annu. Rev. Microbiol. (2001) 55:453-483.

[52] Sacks D., Lawyer P., Kamhawi S., The biology of Leishmania-sandfly interactions, in: Myler P.J., Fasel N. (Eds.), Leishmania after the genome, Chapter 10, Caister Academic Press, 2007, pp. 205-238.

[53] Sant'Anna M.R., Alexander B., Bates P.A., Dillon R.J., Gene silencing in phlebotomine sand flies: xanthine dehydrogenase knock down by dsRNA microinjections, Insect. Biochem. Mol. Biol. (2008) 38:652-660.

[54] Schlein Y., Jacobson R.L., Messer G., Leishmania infections damage the feeding mechanism of the sandfly vector and implement parasite transmission by bite, Proc. Natl. Acad. Sci. USA (1992) 89:99449948.

[55] Schröder J.M., Reich K., Kabashima K., Liu F.T., Romani N., Metz M., et al., Who is really in control of skin immunity under physiological circumstances - lymphocytes, dendritic cells or keratinocytes? Exp. Dermatol. (2006) 11:913-929.

[56] Shortt H., Swaminath C., The method of feeding of Phlebotomus arpentipes with relation to its bearing on the transmission of kala azar, Indian J. Med. Res. (1928) 15:827-836.

[57] Smith D.F., Peacock C.S., Cruz A.K., Comparative genomics: from genotype to disease phenotype in the leishmaniases, Int. J. Parasitol. (2007) 37: 1173-1186.

[58] Stierhof Y.D., Bates P.A., Jacobson R.L., Rogers M.E., Schlein Y., Handman E., Ilg T., Filamentous proteophosphoglycan secreted by Leishmania promastigotes forms gel-like three-dimensional networks that obstruct the digestive tract of infected sandfly vectors, Eur. J. Cell Biol. (1999) 78:675-689.

[59] Svobodová M., Votýpka J., Experimental transmission of Leishmania tropica to hamsters and mice by the bite of Phlebotomus sergenti, Microbes Infect. (2003) 5:471-474.

[60] Svobodová M., Volf P., Votýpka J., Experimental transmission of Leishmania tropica to hyraxes (Procavia capensis) by the bite of Phlebotomus arabicus, Microbes Infect. (2006) 8:1691-1694.

[61] Tripp C.H., Haid B., Flacher V., Sixt M., Peter H., Farkas J., et al., The lymph vessel network in mouse skin visualised with antibodies against the hyaluronan receptor LYVE-1, Immunobiology (2008) 213:693-916.

[62] Volf P., Hajmova M., Sadlova J., Votýpka J., Blocked stomodeal valve of the insect vector: Similar mechanism of transmission in two trypanosomatid models, Int. J. Parasitol. (2004) 34:1221-1227.

[63] Walters L.L., Modi G.B., Chaplin G.L., Tesh R.B., Ultrastructural development of Leishmania chagasi in its vector, Lutzomyia longipalpis (Diptera: Psychodidae), Am. J. Trop. Med. Hyg. (1989) 41:295-317.

[64] Walters L.L., Leishmania differentiation in natural and unnatural sand fly hosts, J. Eukaryot. Microbiol. (1993) 40:196-206.

[65] Warburg A., Schlein Y., The effect of postbloodmeal nutrition of Phlebotomus papatasi on the transmission of Leishmania major, Am. J. Trop. Med. Hyg. (1986) 35:926-930.

[66] Warburg A., The structure of the female sand fly (Phlebotomus papatasi) alimentay canal, Trans. R. Soc. Trop. Med. Hyg. (2008) 102:161-166.

[67] Wohlfert E., Belkaid Y., Role of endogenous and induced regulatory $\mathrm{T}$ cells during infections, J. Clin. Immunol. (2008) 28:707-715. 